\title{
The flow properties of foam
}

\section{A B J Kroezen, J Groot Wassink and C A C Schipper}

Twente University of Technology. Enschede. The Netherlands

\begin{abstract}
The rheological behaviour of foam, prepared from an aqueous solution with lauryl sulphate as surfactant, has been measured with a viscometer as a function of the blow ratio, bubble diameter distribution and concentration of the thickener used. The influence of wall slip and of hysteresis effects on the rheological behaviour have been determined. Calculations and measurements pertaining to the pressure drop in pipes have been performed, in which the foam was considered as a compressible pseudoplastic whose rheology depended on blow ratio. The distribution of the foam passing through a closed distribution unit has been studied. This distribution unit consisted of a pipe and a cross-head distribution slit. Complementary measurements of the flow of foam through narrow slits have been made. The design of a closed foam distribution unit is discussed. The consequences of the choice of several design parameters are examined.
\end{abstract}

\section{INTRODUCTION}

In the process of foam application, in which the foam is used as a medium for transport of chemicals onto a substrate, the application results as well as the design of the application unit are dependent to a great extent on the rheological properties of the foam.

A foam application unit can be considered to consist of three functional parts, i.e. the generation of the foam, its transport and distribution, and its application on the substrate. The foamability and foam structure in the mixer are dependent on the rheological properties of the foam $[1,2]$. In the application section the rheological behaviour determines the uniformity of application and the penetration into the substrate. Based on a study of the rheological properties of the foam, the transport and the width distribution of the foam on the substrate will be considered and described in this paper. The distribution depends mainly on the flow properties of the foam. A uniform width distribution is achieved by taking flow behaviour into account and designing a suitable layout for the distribution unit, especially in closed distribution units.

Even though several authors have previously examined the flow properties of foam [3-5], a close examination has been carried out in order to arrive at a complete description of foam hydrodynamics. Flow behaviour has been measured as a function of blow ratio, bubble diameter distribution and the viscosity of the liquid phase. In particular the effects of wall slip and decrease in bubble diameter as a result of applied shear stress have been studied. Flow through pipes and through a foam distribution unit has also been examined in detail. In the commercial foam distribution unit used, the foam passed through a dead-end pipe, with a long narrow cross-head slit. The description of the flow of foam through such a distribution unit is based on a combination of the description of flow through pipes and flow through narrow slits [6]. In particular the distributing properties of such a unit, and the manner in which the design parameters influence those properties, have been considered more closely. The study of the flow of foam in such a distribution unit has been performed with a thickened liquid system, in which the viscosity corresponded to the range of viscosities of the liquids used in the foam printing of textiles [6]. In this process especially, high demands are made on the uniformity of application.

\section{RHEOLOGICAL PROPERTIES OF FOAM}

Foams are defined as colloidal gas emulsions, with the gas representing the dispersed phase and the liquid the continuous phase; the gas phase is distributed throughout the liquid matrix. The structure of the foam may be described either by the blow ratio or by the density of the foam combined with the bubble diameter distribution. Foams have unstable structures that continuously change with time. The structural changes proceed by the following mechanisms of destabilisation [8-10], which occur because of drainage of the liquid present in the lamellae and the plateau borders. The result is a local change in the gas-liquid ratio. The bubble diameter distribution of the foam is also subject to continuous change, primarily due to gas diffusion from bubbles with a large internal pressure (small bubbles) to those with a smaller internal pressure (larger bubbles). This causes small bubbles to become smaller, and large bubbles to grow larger. The bubble diameter distribution also changes as a result of ruptured lamellae, causing bubbles to coalesce.

Foams are compressible media with rather large viscosities. They are usually described by means of a pseudoplastic model (Eqn 1) or by a model pertaining to a Bingham plastic (Eqn 2) [3,4]:

$$
\begin{gathered}
\tau=k(\mathrm{~d} u / \mathrm{d} x)^{n} \\
\tau=\tau_{0}+k(\mathrm{~d} v / \mathrm{d} x)^{n}
\end{gathered}
$$

The viscosity is dependent on the structure of the foam and increases at decreasing bubble diameter and decreasing foam density $[3,5]$. For foams with a relatively low density an applied stress lower than the yield stress results in elastic behaviour.

Foams are not perfect homogeneous media, even when the diameter of the flow opening is only a few times that of the mean bubble diameter. Another aspect to the inhomogeneity of the foam is a wall slip velocity along a 
smooth surface $[3,4,11]$. Since the viscosity of the foam is many times greater than the viscosity of the liquid, an appreciable velocity gradient may exist over a thin liquid layer, which adheres to a smooth wall.

\section{Experimental procedures}

The rheological properties of a foamed aqueous solution containing $10 \mathrm{~g} / 1$ lauryl sulphate and different concentrations of the thickener Solvitose FN (a starch ether) were determined using a coaxial Brabender viscometer. The foam was produced in a rotor-stator mixer [1]. The diameter of the cylinder of the viscometer was about $5 \mathrm{~cm}$, and the free annular space between the inner and outer cylinders, which is taken up by the liquid, could be varied from 0.75 to $3.0 \mathrm{~mm}$ wide. The viscometer could reach shear rates in the range $0.5-1800 \mathrm{~s}^{-1}$ and a maximum shear stress of $450 \mathrm{~N} / \mathrm{m}^{2}$. The rotational speed could be increased or decreased continuously by means of a programmed rotational speed controller.

In the viscometer continuous axial flow could be achieved by letting the foam flow into the annular space from the bottom up. In this particular construction it is possible to supply continuously the annular space with foam just produced, thus minimising the chance of taking measurements on destabilised foam. The continuous flow is stopped only during the actual measurement, so that it will not influence the measurement. The construction has proved useful, especially for non-thickened foams, since destabilisation due to drainage proceeds rather rapidly for these foams. For measurements on systems with higher viscosities, the annular space was not continuously supplied with fresh foam but was filled only once.

To examine wall slip, use was made of smooth stainless steel cylinders, and of cylinders with a profiled surface which stops slip from occurring $[3,12]$. The profiled surface was achieved by lining both the inner and outer cylinders with plastic gauze having a mesh of $1 \mathrm{~mm}$ and a thickness of $350 \mu \mathrm{m}$. The observed differences in the results, measuring with and without gauze, can be ascribed to slip effects.

The rheology of the foam as a function of bubble diameter distribution was examined as follows. The outer cylinder was replaced by a perspex cylinder so that the actual bubble diameter distribution, as it existed during the course of measurement, could be photographed. For the measurements where the perspex cylinder was not used. prior to taking the viscosity measurement the bubble diameter distribution was allowed to assume the stable value found in the viscometer at shear rates of 1000 or $1800 \mathrm{~s}^{1}$. The reproducibility of a known bubble diameter distribution of a given foam was thus assured. An additional difficulty appeared when the rheological behaviour of highly viscous foam was to be determined. The viscoelastic behaviour of those foams gives them a tendency to creep along the inner cylinder, so that a zone appeared in the annular space in which the foam contact between the inner and outer cylinders had been lost during measurement.

In Table 1 the different liquid systems which were used for measurements are given

\section{Experimental results}

For the LS-20, LS-30 and LS-40 systems, where the
TABLE 1

\section{Liquid systems used (all contained $10 \mathrm{~g} / 1$ lauryl sulphate)}
Code
Solvitose FN concn (g/l)

\section{LS-0}

LS-20

20

LS-30

LS-40

40

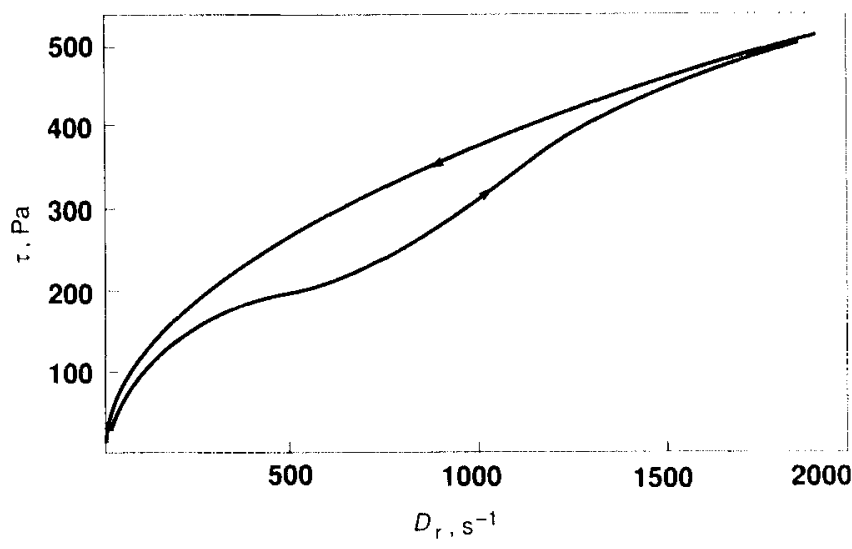

Figure 1 - Foam rheology measurement for system LS-30 (density $200 \mathrm{~g} /$ )

annular space was filled with foam, the rheological curve was measured by allowing the rotational speed to increase continuously from zero to $500 \mathrm{rev} . / \mathrm{min}$ (shear rate 1800 $\mathrm{s}^{-1}$ ), with an acceleration of $200 \mathrm{rev} . / \mathrm{min}^{2}$. The rotational speed was then decreased with a similar magnitude of deceleration. In this way the rheological curve could be registered on the recorder. Any hysteresis effect appearing was thus recorded simultaneously. An important hysteresis effect observed is shown in Figure 1.

Under the influence of shear the foam becomes more viscous. A closer analysis of the experimental results showed that the cause of the hysteresis was a change in the bubble diameter distribution. At a shear rate of $600 \mathrm{~s}^{\prime}$ in our example a decrease of the bubble diameter occurred. which continued until the maximum shear rate was reached. The foam maintained its decreased bubble diameter on returning from maximum shear rate to a shear rate of zero. The difference in the rheology of the foam as described by the two different paths of the curve is thus caused on the one hand by the original foam having a larger bubble diameter, and on the other by the foam with the smaller bubble diameter. A larger viscosity in combination with a smaller bubble diameter is in agree ment with earlier findings [3]. A decrease in bubble diameter at higher shear rates for flow through pipes and during viscosity measurements has also been verified by other authors [13,14].

Trauter and Vialon [5] have published curves of viscos ity as a function of shear rate that are similar to that shown in Figure 1. Their experiments were carried out primarily on highly viscous foams at low density, and showed that the progressive rise in viscosity was immediately followed 
by a disturbance of the flow and a loss of contact between the inner and outer cylinders. This interference was also observed in our measurements, and seems to be the result of the strong viscoelastic properties of the foam. These properties result in normal tension in the direction towards the inner cylinder, and thus causes the foam to creep upwards along the inner cylinder wall. This is known as the Weisenberg effect. Through this phenomenon the contact between the inner and outer cylinder may be lost entirely. At higher foam densities the disturbance of the flow is less important and the decrease of the bubble diameter could be registered unambiguously, correspond ing to the flow curve in Figure 1.

The increase in viscosity shown in the flow curves of Trauter and Vialon were, however, not interpreted in terms of changes occurring in the bubble diameter distribution

The hysteresis effect is determined by the increase in bubble diameter caused by gas diffusion, and by a decrease in the bubble diameter as a result of shear. The increase in bubble diameter due to gas diffusion has been extensively reported in literature, and will not be considered in detail in this paper $[9,10]$. However, the decrease in the bubble diameter of foam because of shear has not previously been examined as extensively, even though it is a much faster process, and so we examined it in more detail.

Using a perspex outer cylinder in the viscometer, the decrease in bubble diameter was studied by photographically recording the bubble distribution, using a foam with a relatively large mean bubble diameter, which was subjected to shear for about $10 \mathrm{~s}$. The decrease in the mean bubble diameter can be described in a similar way to that in the rotor-stator foam mixer, as previously discussed [2]. In that paper the bubble diameter distribution characterised by the quantity $d+$ (the mean bubble diameter plus standard deviation) was described by the critical Weber number, as given in Eqn 3:

$$
(W e)=\frac{\tau d+}{\sigma}
$$

The critical Weber number gives the equilibrium bubble diameter $d+$ as a function of applied shear stress and surface tension. In Figure 2 measured values of the LS-30 system with a foam density of about $200 \mathrm{~g} / \mathrm{l}$ are given.

The calculated critical Weber number was 0.48 . This value is $20 \%$ smaller than that calculated from measurements on the rotor-stator mixer, i.e. 0.6 [2]. A possible cause of the difference between the two values may be the difference in process conditions, experimental set-up and liquid system used.

The rheological curves of the foams were measured for foams whose bubble diameters were first reduced to the equilibrium bubble diameter by applying a particular shear stress in the viscometer. This way of measurement is much more accurate than directly measuring foam, whose structure has already been determined prior to introducing it into the viscometer [3]. The rheological curves were characterised using the power law function (Eqn 1). This equation can also be written as shown in Eqn 4 , where the reference shear rate is given the term by $D_{\mathrm{r}, 0}$. The apparent viscosity is given by Eqn 5 . The apparent viscosity $\eta$ is defined at a given shear rate.

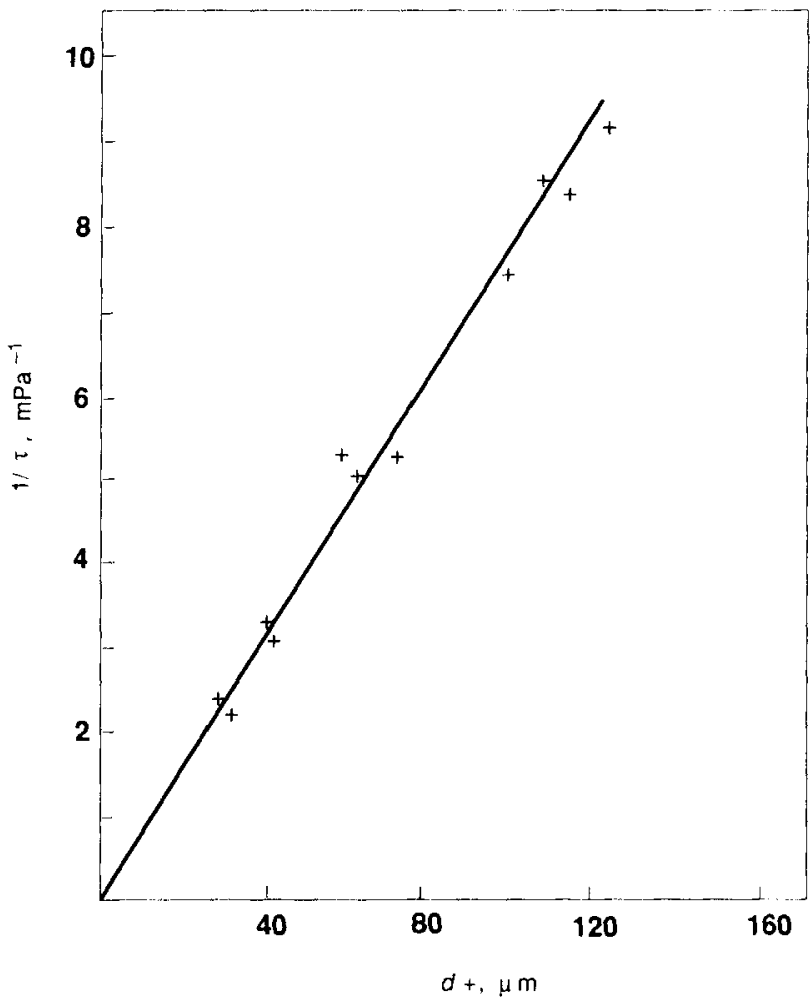

Figure 2-Decrease in bubble diameter in the viscometer

$$
\begin{gathered}
\tau=k_{2}\left(D_{\mathrm{r}} / D_{\mathrm{r}, 0}\right)^{n} \\
\eta\left(D_{\mathrm{r}}\right)=k D_{\mathrm{r}}^{n-1}=\frac{k_{2}\left(D_{\mathrm{r}} / D_{\mathrm{r} .0}\right)^{n}}{D_{\mathrm{r}}}
\end{gathered}
$$

By choosing the reference shear rate $D_{r .0}$ in the middle of the range of values applied, $k_{2}$ can be determined more accurately than can a $k$ value, which is by definition equal to the apparent viscosity at a shear rate of $1 \mathrm{~s}^{-1}$.

The apparent viscosity of foams of the LS-30 system is given in Figure 3 as a function of the foam density and bubble diameter distribution. The apparent viscosity increases as foam density decreases; the influence of bubble diameter distribution only becomes important at densities lower than $400 \mathrm{~g} /$. At densities lower than this the influence of bubble diameter distribution becomes important when the bubbles begin to resist each other during flow, where they should be able to deform in order to be able to pass one another. The bubbles in foam with a smaller mean bubble diameter are more difficult to deform, due to the higher internal pressure. This effect causes foam with a smaller mean bubble diameter to have a higher viscosity.

In Figure 4 the plasticity factor $n$, which describes the dependence of shear rate on viscosity, is given for the LS-30 system and for shear rates of 10 to $1000 \mathrm{~s}^{-1}$. This factor seemed to be nearly independent of the bubble diameter distribution, so that only the distribution is given as a function of the liquid system used and the foam density.

Measurements on the unthickened LS-0 system were carried out on foams with a density of 30 to $240 \mathrm{~g} / \mathrm{l}$. Difficulty was experienced with these measurements for two reasons. Drainage of the unthickened system occurred rather rapidly, and slip flow of the foam over a thin 


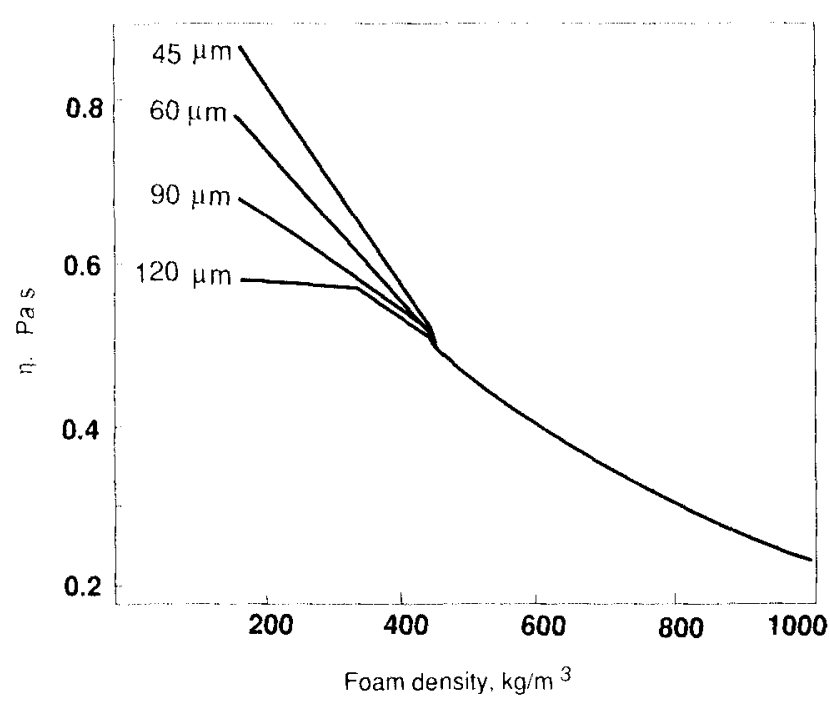

Figure 3 - Foam viscosity in the LS-30 foam system as a function of bubble diameter and foam density

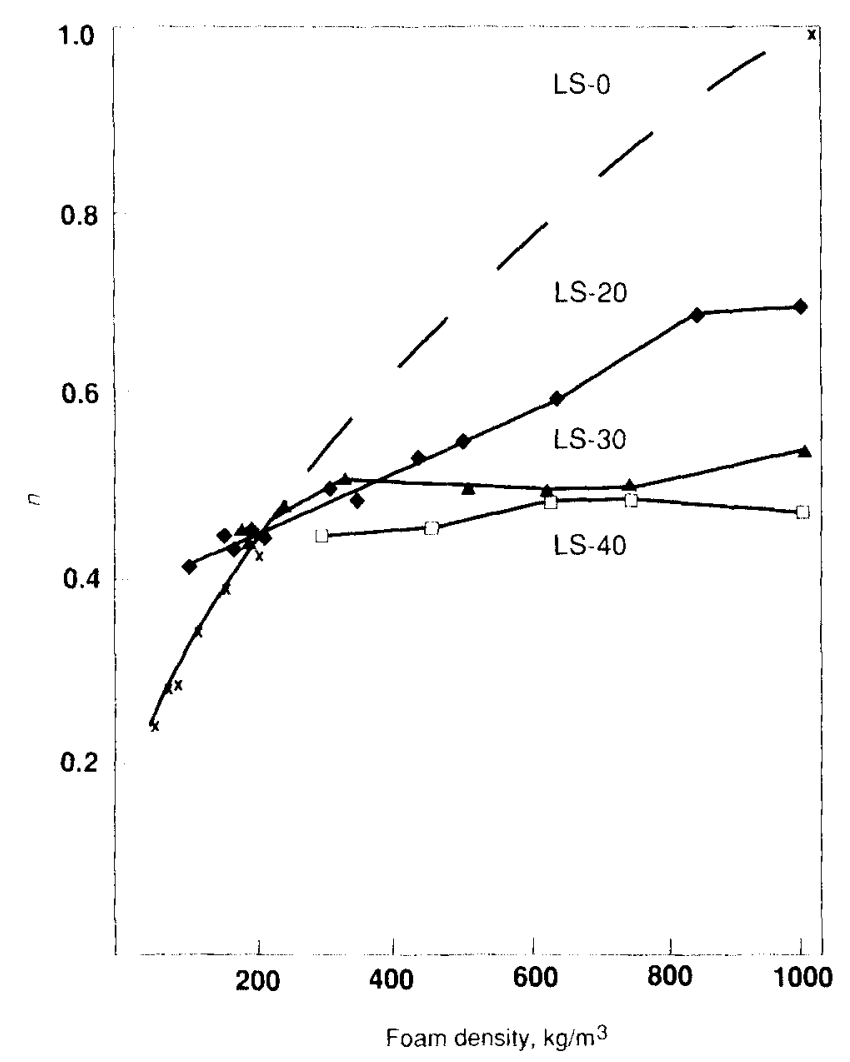

Figure 4-Plots of foam n factors

liquid layer near the smooth cylinder wall could not be neglected. No measurements were carried out on foams with a density greater than $240 \mathrm{~g} / \mathrm{l}$, since drainage of the foam proceeded so rapidly that the results were not reliable. The experimental procedure for these foams was as follows. Between each reading the annular space was slowly rinsed with foam just produced, whereby the diameter of the foam bubble was simultaneously decreased to a stable value at a shear rate of $1000 \mathrm{~s}^{1}$. Just prior to taking a reading the flow through the annular space was stopped, and the shear rate at which the reading was to be taken was set. The measurements were performed with two cylinders, both having a profiled surface. Measurements were also done with the inner cylinder having a profiled surface, and the outer cylinder a smooth surface, in order to determine the contribution of the slip flow along a smooth surface. The rheological curve thus obtained is composed of two components: slip flow and shear in the foam. A typical rheological curve with and without slip flow is given in Figure 5, showing the importance of slip flow at low shear rates.

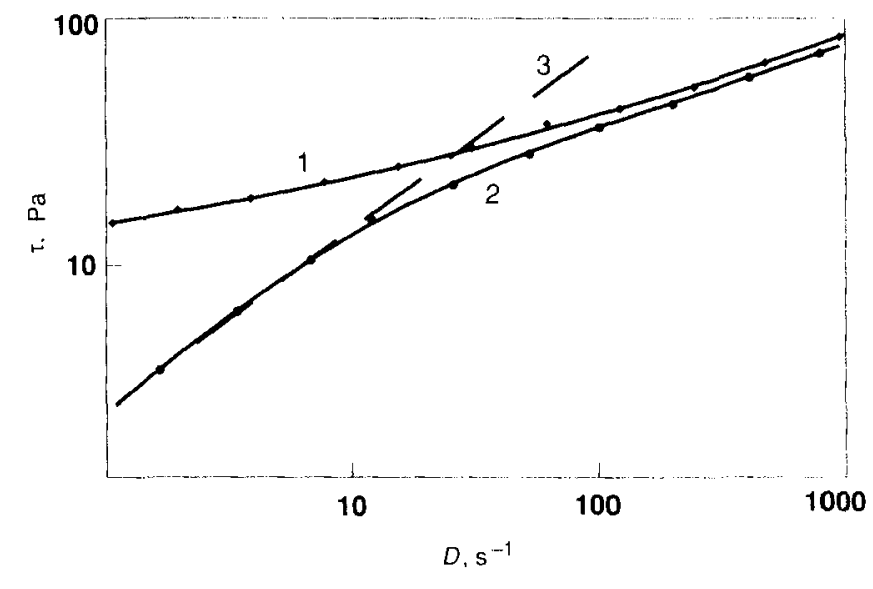

1 - Rheological curve, toam (measured)

2 - Rheological curve, foam + slip (measured)

3 - Contribution of slip (calculated)

Figure 5-Rheological curve with and without slip for system LS.O (density $75 \mathrm{~g} /$ )

The contribution of slip flow (Eqn 6) is appreciable for dispersions in which the difference between the viscosity of the dispersion and the viscosity of the continuous phase is very large:

$$
\tau=a(\Delta v)^{b}
$$

In Eqn 6 slip velocity is related to the shear stress applied. Since the slip velocity gradient exists over a thin liquid boundary layer, it is possible to calculate a mean boundary thickness based on the known liquid viscosity (Eqn 7):

$$
\tau=\eta_{1} \frac{\Delta v_{s}}{\delta_{e}}
$$

For values of $b$ not equal to 1 , the thickness of the boundary layer will change as a function of slip velocity (Eqn 8):

$$
\delta_{e}=\eta_{1} \frac{\Delta u_{s}^{I-b}}{a}
$$

The contribution of slip flow is shown in Figure 6, curve 3 (which is the difference between the curves 1 and 2). The constants $a$ and $b$, as measured, are given. In Table 2 several values for $a$ and $b$, as measured, are given. A value of 0.75 for $b$ indicates that the effective thickness of the boundary layer, over which shear is taking place, increases with increasing shear stress. The thickness of the bound ary layer has been calculated for two apparent shear rates in the viscometer, making use of Eqn 8 . When comparing this value with an estimated value for the mean lamellar 
thickness, it seems that the thickness of the boundary layer is relatively small. The mean lamellar thickness can be calculated from Eqn 9 [4]:

$$
\delta_{\mathrm{t}}=\frac{d \rho_{\mathrm{s}}}{3 \rho}
$$

The thickness of the boundary layer $\delta_{p}$ is less than found by other authors $[3,4]$. One of the possible causes may be that the bubble diameter in our experiments was smaller. Since the measured mean thickness of the boundary layer is smaller than that calculated, it follows that there is no transport of liquid from the bulk of the foam to the boundary layer.

TABLE 2

\section{Slip flow data for system LS-0}

\begin{tabular}{rccccr}
$\rho_{s}$ & $a$ & $b$ & \multicolumn{2}{c}{$\delta_{e}\left(\times 10^{-7}\right)$} & \begin{tabular}{c}
$\delta_{\mathrm{r}}$ \\
\cline { 5 - 6 }
\end{tabular} \\
\hline 33 & 423 & 0.75 & 5 & 11 & 8 \\
55 & 314 & 0.75 & 6 & 17 & 160 \\
75 & 266 & 0.75 & 8 & 19 & 250 \\
95 & 244 & 0.75 & 9 & 21 & 350 \\
135 & 196 & 0.75 & 10 & 250 & 560 \\
190 & 256 & 0.95 & 28 & 330 & 940
\end{tabular}

The contribution of slip becomes especially important at low shear rates. This is a result of the fact that the ratio of the apparent viscosity of the foam and the viscosity of the liquid is quite large at low shear rates, even though this ratio decreases rapidly with increasing shear rates.

A summary of the rheological curves obtained for shear rates ranging from 50 to $1800 \mathrm{~s}^{1}$ and for the different foam systems is given in Figures 4 and 6 respectively. In Figure 6 the apparent viscosity at a shear rate of $800 \mathrm{~s}^{-1}$ is plotted as a function of foam density for four different liquid systems. These measurements were always taken after the bubble diameter had been decreased at a shear rate of $1800 \mathrm{~s}^{\prime}$ for the LS-20, LS-30 and LS-40 systems, and at a shear rate of $1000 \mathrm{~s}^{-1}$ for the LS-0 system.

The viscosities of the foams in the different liquid systems depend strongly on liquid viscosity. In general, the foam viscosity can be considered to consist of a structural viscosity component and of the viscosity of the pure liquid. The plasticity factor $n$ of the various liquids differ considerably, but at a density of $200 \mathrm{~g} / \mathrm{l}$, for example. all foams measured seemed to have plasticity factors of about 0.45 .

\section{RHEOLOGY AND PRESSURE DROP IN FOAM FLOW}

\section{Pressure drop over pipes}

If the rheology of a foam is known, the pressure drop over a length of pipe can be calculated. The rheology of foam can be described by the power law within a certain range of shear rates (Eqn 1). Integration of this equation for flow

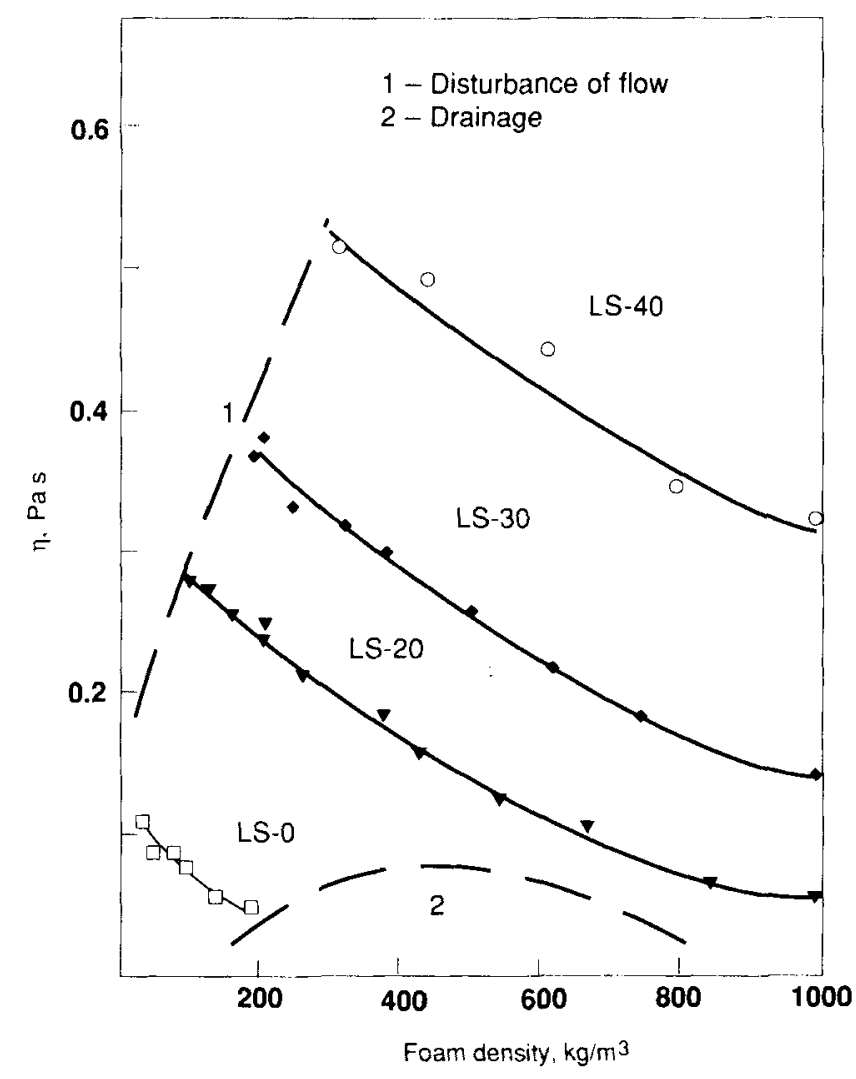

Figure 6-Plots of foam viscosity

through a length of pipe yields Eqn 10 , which gives the pressure drop over a unit length as a function of the flow rate:

$$
\frac{\mathrm{d} P}{\mathrm{~d} x}=F_{v}^{n}\left(\frac{3 n+1}{\pi n}\right)^{n} R^{-(3 n+1)} 2 k
$$

When calculating the pressure drop, however, the compressibility of the foam cannot be neglected. Since the foam expands over a certain length of pipe, the flow rate and the rheology determined by the foam structure change in the direction of flow. The local foam density and the bubble diameter distribution change as function of the space coordinates. The compressibility of the foam may be calculated by making use of the ideal gas law when describing the gas phase, and considering the liquid to be an incompressible medium. The actual foam density follows from Eqn 11:

$$
\rho_{\mathrm{s}}=\frac{\rho_{\mathrm{g}}}{1+B_{\mathrm{O}} P_{\alpha} / P}
$$

The change in bubble diameter can be calculated using Eqn 12:

$$
d_{P}=d_{0}\left(P_{O} / P\right)^{1 / 3}
$$

The power of $1 / 3$ present in Eqn 12 shows that bubble diameter changes only slightly in comparison with flow rate and foam density.

Only for the LS-30 system were all the parameters measured, so that further calculations are valid for this system only. 
The pressure drop over a pipe can thus be calculated with Eqns 10 and 13-17:

$$
\begin{gathered}
F_{\mathrm{v}}=F_{\mathrm{m}}\left(1+B_{\circ} P_{\mathrm{o}} / P\right) \\
n=\mathrm{f}(P) \\
k=\mathrm{f}(P)
\end{gathered}
$$

Initial condition

$$
\begin{gathered}
F_{\mathrm{m}}=\text { constant } \\
X=L \quad P=P_{0}
\end{gathered}
$$

Calculations were carried out on the LS-30 foam system, where the foam density was $200 \mathrm{~g} / 1$ at a pressure of one atmosphere. The bubble diameter $d+$ of this system was $60 \mu \mathrm{m}$.

The calculations were verified by means of pressure drop measurements over pipes with a diameter of 1 to 1.5 $\mathrm{cm}$ and a length of $8 \mathrm{~m}$, in which the flow varied from 0.5 to $1.5 \times 10^{-6} \mathrm{~m}^{3} / \mathrm{s}$. The pressure drop increased to a maximum of $4 \mathrm{~atm}$. A good agreement was found between calculated pressure drops (Eqns 11-14) and measured pressure drops. The change in the pressure drop per unit length of pipe, at a total pressure drop increasing to $4 \mathrm{~atm}$, was so large that, if the compressibility and the changing rheology had been neglected, a large discrepancy between calculated and measured values would have been found.

The influence of changing bubble diameter on rheological properties during the expansion of the foam was very small. Therefore the pressure dependence of the rheology of the foam in Eqns 4 and 5 may be ascribed to a changing foam density.

\section{Flow of foam through narrow slits}

For the calculation of flow through slits Eqn 18 is substituted for Eqn 10:

$$
\frac{\mathrm{d} P}{\mathrm{~d} z}=\left(\frac{F_{\mathrm{V}}}{y}\right)^{n}\left(\frac{2 n+1}{2 n}\right)^{n}(0.5 h)^{-(2 n+1)} k
$$

In addition the supplementary equations (Eqns 13-15) must be used in order to be enabled the pressure drop over a slit to be calculated numerically. For flow of foam through narrow slits, the foam can no longer be viewed as one continuous medium. This is especially the case when the diameter of the slit, $h$, is less than $1 \mathrm{~mm}$. Since in some distribution units very narrow slits are used, the flow of foam through a narrow slit has been examined in more detail. The LS-30 foam system was used with a foam density of $200 \mathrm{~g} / 1$ under atmospheric pressure. In these experiments the pressure drop as a function of the flow was measured for a number of slit diameters, $h$, and slit lengths, $z$. The flow of foam was varied from 0.02 to 2 $\mathrm{kg} / \mathrm{min}$. The experimental set-up consisted of a foam feed pipe connected to a slit having an adjustable diameter and

\begin{tabular}{|c|c|c|c|c|c|c|}
\hline \multirow{2}{*}{$\begin{array}{l}\text { Length } \\
\text { (mm) }\end{array}$} & \multicolumn{6}{|c|}{ Diameter (mm) } \\
\hline & 0.1 & 0.2 & 0.3 & 0.4 & 0.5 & 1.0 \\
\hline 2.5 & + & + & + & & & \\
\hline 5 & + & + & + & + & & \\
\hline 10 & + & + & + & + & + & \\
\hline 25 & & + & + & + & + & + \\
\hline 50 & & + & + & + & + & + \\
\hline
\end{tabular}
length, while the width of the slit, $y$, was maintained at 5 $\mathrm{cm}$. The dimensions of the slit, that have been used in the experiments are given in Table 3

TABLE 3

Slit flow experiments with system LS-30(a)

(a) Density $200 \mathrm{~g} / \mathrm{l}, d+60 \mu \mathrm{m}$

When using a slit narrower than $1 \mathrm{~mm}$, a substantial deviation was found between the experimental results and the pressure drop calculated from Eqn 18. For slits at lower slit diameters, the measured pressure drop became increasingly lower than the calculated values. The decrease in the measured viscosity of the foam flowing through slits must be ascribed to the fact that the foam can no longer be regarded as a continuous medium, and so a correction factor must be introduced in the equation for calculating foam rheology.

On the evidence provided by the experiments, the decrease in the apparent viscosity of the foam could not be ascribed to wall slip effects. Such an evaluation would lead to the slip velocity being dependent not only on shear stress at the wall, but also on the diameter of the slit. From the results it follows that the reduced pressure drop must be due to a decrease in the viscosity of the bulk of the medium. The correction term introduced to describe adequately the rheological behaviour of foam flowing through a narrow slit is given in Eqn 19:

$$
\begin{gathered}
D_{\mathrm{r}}=\frac{\delta v}{\delta x}=(1 / k \tau)^{1 / n}+g\left(\frac{\delta \tau}{\delta x}\right)^{1 / m} \\
\begin{array}{c}
\text { Power } \\
\text { law }
\end{array} \\
\begin{array}{c}
\text { Correction } \\
\text { term }
\end{array}
\end{gathered}
$$

The correction term is a function of the change in shear stress per unit width in the slit. It is determined by the two effects described below, which cause a viscosity decrease.

Firstly, if the ratio between bubble diameter and slit diameter is large, the actual profiles of velocity and shear rate are discontinuous and deviate substantially from the calculated ones, which are based on continuous profiles. The change in the shear stress over a layer of bubbles. which cannot be treated as a continuous medium, will therefore determine the extent of the error present when calculating the pressure drop over the slit, assuming the foam is a continuous medium.

The second effect is the result of a difference in the 
rotational speed of the bubbles over the width of the slit. In the foam the bubbles will rotate when subject to a certain shear stress as a result of the torque applied on the bubbles. In a lamella the direction of motion of one bubble is in the opposite direction to the motion of a neighbouring bubble. In a monodisperse foam all the bubbles will have the same rotational speed when subject to a constant shear stress. However, when the shear stress gradient is large over a few layers of bubbles, the rotational speeds of the bubbles will differ from each other, resulting in an apparent decrease in viscosity.

The correction term was determined from the experimental results as follows. For the measured pressure drops (and as a function of the dimensions of the slit) the experimental flow rate was compared with the flow rate calculated from Eqn 1 (taking compressibility into account). The difference between these two flow rates $F_{v}^{*}$ follows also from the calculation based on Eqn 18 and the integrated form of Eqn 19 (Eqn 20):

$$
F_{\mathrm{v}}^{*}=g\left(\frac{\delta P}{\delta z}\right)^{1 / m}(0.5 h)^{2}
$$

In the calculations mean values over the slit of $\delta P / \delta z$ and $F_{\mathrm{v}}{ }^{*}$ were taken, the terms $m$ and $g$ being assumed to be independent of the changing foam structure over the slit. The values thus determined for $g$ and $m$ were $3.49 x$ $10^{-9}$ and 0.55 respectively. These values are valid for a foam with a density of $200 \mathrm{~g} / \mathrm{l}$ under atmospheric conditions and a mean bubble diameter of about $40 \mu \mathrm{m}$.

With Eqn 19 an accurate description can be made when the diameter of the slit lies between 0.2 and $1 \mathrm{~mm}$. At a diameter of $0.11 \mathrm{~mm}$ a deviation was found from Eqn 19 which amounted to $20 \%$ of the calculated value. At slit diameters $0.2,0.3,0.4,0.5$ and $1.0 \mathrm{~mm}$ the difference between the calculated and the measured values lay well within $10 \%$

\section{Design of a distribution unit}

The commercial foam distribution unit used in this work consists of a feed pipe with a drain along the side. The drain is formed by a slit of a certain diameter and length. The dimensions of the slit are chosen to maintain an even distribution over the entire drain length. Since a pressure drop builds up over the feed pipe, the pressure drop in the direction of the length of the pipe also unavoidably changes. In order in such a case to be able to achieve even drainage from the slit, its dimensions must be adjusted over the entire length of the pipe. A choice can be made in the design between a non-constant slit length or a nonconstant slit diameter. Figure 7(a) shows a single distribution pipe; a commercially available layout consists of two distribution pipes having a slit with a non-constant diameter. The distribution pipes are mounted so that flows in them is in opposite directions [7]. The errors arising from the drain in each slit cancel each other out. This principle is sketched in Figure 7(b).

The distribution unit as shown in Figure 7(a) is used in polymer technology for the extrusion of polymer sheetings, flat films and blown films. The flow of a pseudoplastic through a cross-head slit has been discussed by many authors $[6,15]$. By describing the rheology with the power law (Eqn 5) it becomes possible to calculate the flow through the distribution unit. The conditions that dictate the change in the slit geometry can also be calculated [15]. It may be concluded that the distribution strongly depends on the plasticity factor $n$ and the geometry of the distribution unit. When the $n$ factor and the geometry remain constant, the rheological constant $k$ and the flow are of no influence on the uniformity of the distribution.

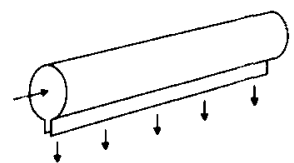

(a)

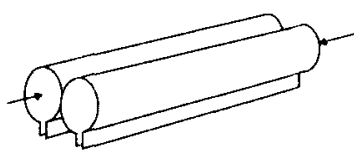

(b)
Figure 7 - Foam distribution units: (a) single, (b) double

The magnitude of the deviation of a proportional distribution can be expressed as a uniformity index (UI). This index is defined as the ratio of the difference between the maximum and minimum flow leaving the unit over the entire width, and the mean flow leaving the unit.

The same type of distribution unit for foams leads to quite different conclusions with regard to the influence of the relevant variables on foam distribution. It is possible to describe foams as being pseudoplastic with a power law rheology, but they still have specific properties such as compressibility and the heterogeneous character of the flow through relatively narrow slits.

It is possible to arrive at a description with which the consequences of a particular choice of design parameters on the distribution of the foam can be calculated by combining the models of flow through pipes and flow through slits. In this paper not all the consequences of the choices of the free design parameters are examined. By using an example in which the flow in one single distribution pipe is calculated, it will be shown how a design can be calculated that is capable of distributing the foam evenly. It will then be shown how the magnitude of the flow passing the pipe will affect the distribution.

A design has been made for a distribution pipe based on foam with a flow of $0.16 \mathrm{~kg} / \mathrm{s}$ (LS-30, $200 \mathrm{~g} / \mathrm{l}, d+=60$ $\mu \mathrm{m})$. The foam was distributed over a width of $2 \mathrm{~m}$, the diameter of the feed pipe was $2.5 \mathrm{~cm}$, the length of the slit was $5 \mathrm{~mm}$ (over the entire width of the unit) and the diameter of the slit at the end of the pipe was $0.7 \mathrm{~mm}$. It was calculated how the width of the slit should vary in order to achieve an even distribution over the entire width of the unit. This adjustment is shown in Figure 8(a), and from it the distribution was calculated for three different flow rates. The local ratio $U$, i.e. the ratio between the calculated flow rate leaving the slit and the desired flow rate for an even distribution, is shown in Figure 8(c). The corresponding pressure drops in the distribution pipe are given in Figure $8(b)$.

In this design the foam distribution is strongly dependent on the flow of the foam feed. In a foam printing unit, for example, it is not unusual for large variations in the quantity of foam applied to occur. To achieve an even distribution, where the UI lies between certain values, even when the flow of foam deviates, it is necessary to alter the design. The following changes may be made to give a 


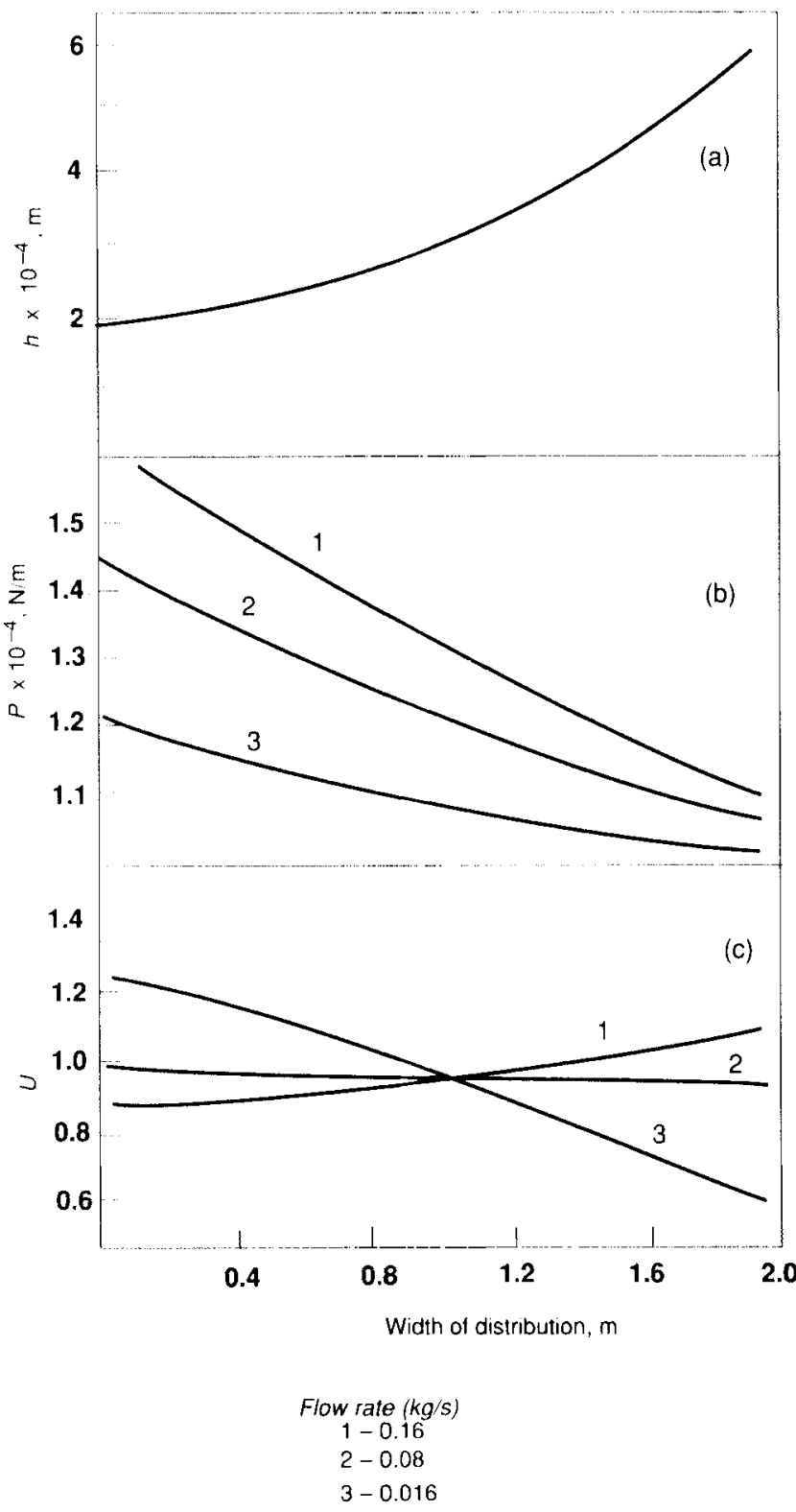

Figure 8 - Typical calculations of foam properties: (a) slit diameter distribution, (b) pressure drop in pipe, (c) uniformity of width distribution

better distribution: a longer slit plate, a larger pipe diameter, or a feed from the middle instead of from the ends of the pipe $[7,15,16]$.

Another method often applied is to use the double distribution pipe. When one was used in the present work, with all other dimensions remaining the same, it was found that the distribution was almost completely independent of the flow.

In summary, it has been shown that once a description of the flow properties of foams is made, this can be used to calculate the foam distribution and optimise the layout of the distribution unit.

\section{REFERENCES}

1. A B J Kroezen and J Groot Wassink, J.S.D.C. 102 (1986) 397

2. A B J Kroezen and J Groot Wassink, J.S.D.C., 103 (1987) 386

3. H G Wenzel. R J Brungaber and T E Stelson, J. Materials, 5 (2) (1970) 396

4. N N Thondavadi, R Lemlich, Ind. Eng. Chem. Proc. Dev., 24 (1985) 748
5. J Trauter and R Vialon, Melliand Textilber. (1985) 571

6. J F Carly, J. Appl. Phys., 25 (1954) 1118.

7. C J Blaak, Melliand Textilber., (1985) 809

8. A Bikerman, 'Foams' (Heidelberg: Springer Verlag, 1973)

9. A J De Vries, PhD thesis (University of Delft, 1957).

10. A Ranadive and R Lemlich, d. Colloid Interfacial. Sci., 70 (2) (1979) 392

11. P Sherman, 'Industrial rheology' (London Academic Press, 1970).

12. D C H Cheng and B R Parker, Proc. 7th International Congress on Rheology $\{19760518$.

13. H G Wenzel, T E Stelson and R J Brungaber, Proc. Amer. Soc. Civil Eng., (Dec 1967) 153

14. M Blackman, Report REM 420. Ministry of Home Security Research and Experiments Department, England (1944).

15. A Bernard, Modern Plastics, (8) (1956) 127

16. J D Jones, Textile Industry, (Oct 1984) 25

\section{LIST OF SYMBOLS}

\section{Symbol Description Unit}

$a$
$b$
$d$
$d+$
$d_{\mathrm{p}}$
$d_{0}$
$D_{\mathrm{r}}$
$D_{\mathrm{r} .0}$
$F_{\mathrm{m}}$
$F_{v}$
$F_{v} / y$

Constant (Eqn 6)

Constant (Eqn 6)

Mean bubble diameter

Mean bubble diameter +

standard deviation

$\mathrm{m}$

Mean bubble diameter at pressure $P$

m

Mean bubble diameter at $P=0$

Shear rate

Reference shear rate

Mass flow rate

Volume flow rate

Volume flow rate per meter width

Constant (Eqn 20)

Slit diameter

Rheological value (Eqn 1)

Rheological value (Eqn 4)

Length of pipe

Constant (Eqn 20)

Plasticity factor (Eqn 1)

Pressure

$\mathrm{N} / \mathrm{m}^{2}$ atm

Radius of tube

$\mathrm{m}$

$U \quad$ Ratio local flow rate and mean flow rate in the slit

(UI) Uniformity index

Velocity

$\mathrm{m} / \mathrm{s}$

$\mathrm{X}$-coordinate

Calculated lamellar

thickness (Eqn 9)

$\mathrm{m}$

$\mathrm{s}^{1}$

$s^{1}$

$\mathrm{kg} / \mathrm{s}$

$\mathrm{m}^{3} / \mathrm{s}$

$\mathrm{m}^{2} / \mathrm{s}$

$\mathrm{m}$

$\mathrm{Pa} \mathrm{s}^{n}$

$\mathrm{Pa}$

$\mathrm{m}$

Thickness of liquid layer

at the wall

Apparent viscosity

$\delta_{e}$

$\eta$

Foam density

Liquid density

$\mathrm{Pa} s$

$\mathrm{kg} / \mathrm{m}^{3}$

$\mathrm{kg} / \mathrm{m}^{3}$

Shear stress

$\mathrm{Pa}$

Yield stress

$\mathrm{Pa}$

Surface tension 